\title{
Modelling realistic ballast shape to study the lateral pull behaviour using GPU computing
}

\author{
François Nader ${ }^{1, *}$, Patrick Pizette ${ }^{2,3}$ Nicolin Govender ${ }^{2,3,4}$ Daniel N. Wilke ${ }^{5}$, and Jean-François Ferellec ${ }^{6}$ \\ ${ }^{1}$ Institut de Recherche Technologique RAILENIUM, F-59300, Famars, France \\ ${ }^{2}$ IMT Lille Douai, Institut Mines-Télécom, Centre for Materials and Processes, F-59000 Lille, France \\ ${ }^{3}$ Univ. Lille, Institut Mines-Télécom, Univ. Artois, Junia, ULR 4515 - LGCgE - Laboratoire de Génie Civil et géoEnvironnement, \\ F-59000 Lille, France \\ ${ }^{4}$ Research Center Pharmaceutical Engineering GmbH, Graz, 8041, Austria \\ ${ }^{5}$ Department of Mechanical and Aeronautical Engineering, University of Pretoria, 0001, South Africa \\ ${ }^{6}$ SNCF Reseau, DGI-VA-CIR, 6 av. F. Mitterrand, 93574 La Plaine St Denis cedex, France
}

\begin{abstract}
The use of the Discrete Element Method to model engineering structures implementing granular materials has proven to be an efficient method to response under various behaviour conditions. However, the computational cost of the simulations increases rapidly, as the number of particles and particle shape complexity increases. An affordable solution to render problems computationally tractable is to use graphical processing units (GPU) for computing. Modern GPUs offer up 10496 compute cores, which allows for a greater parallelisation relative to 32-cores offered by high-end Central Processing Unit (CPU) compute. This study outlines the application of BlazeDEM-GPU, using an RTX 2080Ti GPU (4352 cores), to investigate the influence of the modelling of particle shape on the lateral pull behaviour of granular ballast systems used in railway applications. The idea is to validate the model and show the benefits of simulating non-spherical shapes in future large-scale tests. The algorithm, created to generate the shape of the ballast based on real grain scans, and using polyhedral shape approximations of varying degrees of complexity is shown. The particle size is modelled to scale. A preliminary investigation of the effect of the grain shape is conducted, where a sleeper lateral pull test is carried out in a spherical grains sample, and a cubic grains sample. Preliminary results show that elementary polyhedral shape representations (cubic) recreate some of the characteristic responses in the lateral pull test, such as stick/slip phenomena and force chain distributions, which looks promising for future works on railway simulations. These responses that cannot be recreated with simple spherical grains, unless heuristics are added, which requires additional calibration and approximations. The significant reduction in time when using non-spherical grains also implies that larger granular systems can be investigated.
\end{abstract}

\section{Introduction}

Granular materials are prevalent in civil engineering applications. In railway systems, ballast grains are preferred to support the track (rail/sleeper system), due to its affordability, filtering properties, and established maintenance practices. For high-speed trains, track geometry is of significant importance. Therefore the behaviour of ballast has to be thoroughly understood and accounted for when designing tracks and planning their maintenance.

The Discrete Element Method (DEM) is a useful numerical tool [1-3] that takes into account the medium's discontinuities, characteristics of granular materials at the particle scale. However, DEM is computationally demanding; consequently, individual grain shapes are often simplified as spherical, with heuristics that aim to account for shape effects [4-6].

Fortunately, the Graphical Processing Unit (GPU) compute offers an alternative. The computational benefit of using GPUs allow for shape complexity to be modelled

*e-mail: francois.nader@ railenium.eu

A video is available at https://doi.org/10.48448/h226-bx97 and resolved accurately. The GPU accelerates compute on properly parallelised numerical codes, by allowing simple tasks to be carried out in parallel on thousands of threads. BlazeDEM-GPU simulation framework was one of the first DEM codes to model millions of non-spherical grains with realistic shape approximations in a practical time frame [7-10]. These advancements allow for more complex grain shapes to be simulated, larger-scale simulations to be performed, and multiple simulations to be conducted for sensitivity analysis or design within a tractable computational time frame. For example for railway application, GPU based DEM simulations have been validated to model realistic ballast particle shapes, large number of particles in 3D ballast simulations and ballast using GPU based simulations [11].

In this study, the influence of the approximation detail of realistic grain shapes, obtained from actual ballast scans, on a standard lateral pull test will be studied. The goal is to identify the appropriate level of shape detail to reliably and predictably model the lateral pull test. All simulations are performed using the BlazeDEM-GPU simulation frame- 
work that has been unit tested on three unit test benchmark problems. The unit tests consist of setting up a simple test case with a specific configuration, and validating the numerical results vis-a-vis theoretical or experimental data. BlazeDEM-GPU was tested for an initial overlap between particles, particles bouncing off each other, and particles sliding one on the other.

\section{Numerical method}

The discrete element method $[1,12,13]$ unlike the finite element method, considers a medium as a collection of separate objects, having a set of physical and numerical characteristics. These objects interact with each other through numerically defined contact laws. The contact model used hereafter to simulate ballast grains is a linearspring-dashpot model. This model is characterised by a normal and tangential stiffness coefficients that represent the elastic deformability and dissipative contact interaction, in addition to the dry friction coefficient used in the Amontons-Coulomb's law.

BlazeDEM-GPU is a framework developed by Govender et al. [14], that extends on the shape representation of standard DEM codes in a computationally tractable way by conducting dedicated GPU compute. The contact forces between polyhedral are resolved from the overlap volume between two contacting polyhedra [10], as opposed to simplifying it to the penetration distance [15]. This allows a better description of the contact force evolution [16]. A key feature of this framework is the highly parallelised contact detection algorithm that benefits from the high number of threads available on GPU. It has been applied to obtain insights into mill [17], mixing [18] processes, as well as into backfill response of integral bridge abutments [19] and landslides.

\section{Grain shape}

The behaviour of ballast grains is influenced by the shape of the grains. The angular aspect of the grains' geometry creates a series of interlocking phenomena, that concentrates the loads in specific points of the medium. These loads can sometimes reach magnitudes high enough to break off asperities or even the whole grain into multiple fragments.

The French national railway company Sociéte Nationale des Chemins de Fer Français (SNCF) conducted various studies on ballast grains. An outcome of some of these studies was a library of 3D scanned ballast grains [20,21]. The geometry in raw scan files can sometimes have vertices that are too close together, which complicates the numerical treatment of the grains without necessarily adding to the precision of the model. This is circumvented in an automated fashion by using a script that looks for such vertices and deletes them, recreating a geometry without these vertices, as shown in table 1 . Consider the example ballast depicted in Figure 1, the geometry of a 3D scanned grain before (raw) and after (treated) eliminating the vertices that are too close to others. The modifications are
Table 1. Geometrical features of a ballast grain before and after mesh treatment, as well as spherical and cubic grains

\begin{tabular}{lcccc}
\hline & Vert. & Faces & $\begin{array}{c}\text { Surf. Area } \\
\left(\mathrm{cm}^{2}\right)\end{array}$ & $\begin{array}{c}\text { Vol. } \\
\left(\mathrm{cm}^{3}\right)\end{array}$ \\
\hline Raw & 33 & 63 & 107 & 84 \\
Treated & 13 & 22 & 84.6 & 52 \\
Spheres & - & - & $84^{*}$ & $93^{*}$ \\
Cube & 8 & 6 & 115 & 84 \\
\hline *: average values, multiple sphere sizes are used
\end{tabular}

quantified in Table 1, as well as the preservation of some particle properties. These scans will be used to specify the grain geometry to be used in future simulations. In this study however, only spherical and cubic grains will be used in simulations. Figure 1 shows the used spherical and cubic grains. The table 1 also shows the characteristics of these grains. Note that three sphere sizes are used for spherical grains, the largest sphere being $20 \%$ larger than the smallest sphere. The values shown in table 1 (surface area and volume) are averages. The spheres and cubes are chosen to have the same volume as the raw ballast grain. This constitutes a preliminary step, to justify proceeding to shapes that are closer to that of real grains.
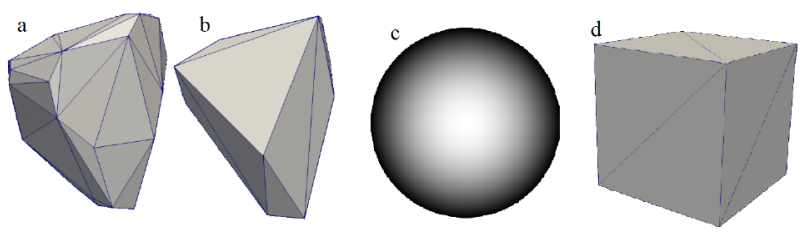

Figure 1. Raw (a) and treated (b) geometry of 3D scanned ballast grain, spherical grain (c), and cubic grain (d)

\section{Lateral pull test simulation}

\subsection{Sample preparation}

In this preliminary investigation, two types of simplified grain shapes are investigated to highlight the effect of grain shape on the lateral pull response. The results presented highlights the importance of moving away from spherical grain shapes to study ballast grains. A sample of spherical grains will be generated, along with an example of cubic grains. The same numerical input parameters are chosen for both granular systems, to isolate only the effect of modelling the grain shape.

A first step in the sample preparation is a gravity deposit of a bed of grains, on top of which is embedded a concrete sleeper (cuboid shape). Figures 2 and 3 show the spherical and cubic granular systems, respectively. Enough time is given for the sleeper, surrounded by a number of layers of grains, to settle under gravity on top of a bed of stable ballast.

BlazeDEM-GPU provides the advantage of simulating the deposit of around 34000 cubic grains in a very reasonable computing time ( 2 hours), using a reasonably priced graphic card (RTX 2080Ti). 


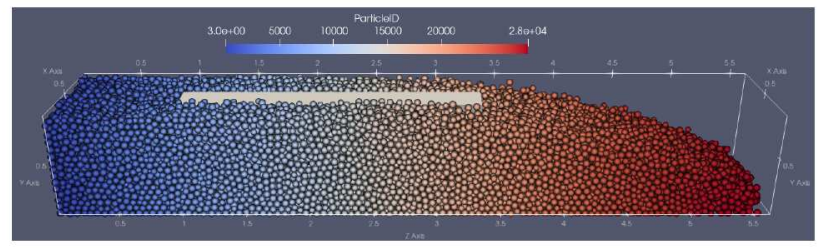

Figure 2. Sample prepared using spherical grains - The color scale indicates the particle ID

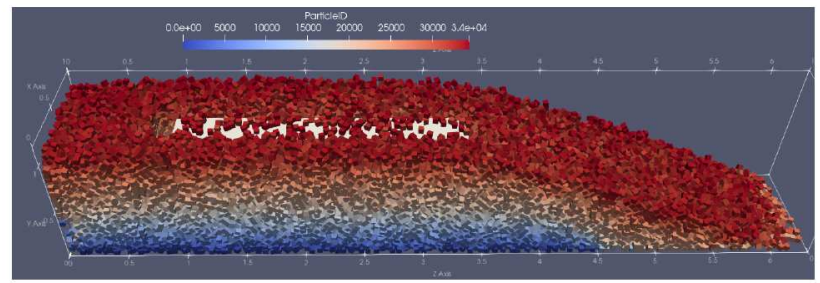

Figure 3. Sample prepared using cubic grains - The color scale indicates the particle ID

\subsection{Lateral pull}

For each case of grain shape, a constant lateral velocity of $2 \mathrm{~cm} / \mathrm{s}$ is applied along the length of the sleeper, to simulate a load pull test, which is a test usually done to predict the behaviour of ballast under loading. The contact of grains with the walls is a dry frictional contact. Figure 4 shows the evolution of the lateral force on the sleeper as it moves, as well as the lateral displacement, with respect to time, for spherical and cubic grains.

A steep increase in the reaction force is observed in the case of spherical grains, and the force reaches its maximum value of around $1750 \mathrm{~N}$ for a displacement of around $10 \mathrm{~cm}$, and keeps fluctuating around that value.

In the case of cubic grains on the other hand, the reaction is generated more gradually. The force keeps climbing with the lateral movement of the sleeper, until it reaches a maximum value of around $2350 \mathrm{~N}$.

Both spherical and cubic grains are subjected to stick/slip phenomena. Grains actually reach a position where movement is not allowed any more, and a high magnitude force is required to free the contact after sticking, and cause a sudden rearrangement. In the case of the cubic grains, the stick slip phenomena take place at the level of the grains' angular corners and edges, so at a higher frequency, which generates a higher resistance to the movement of the sleeper. The delayed response in this case is due to the initial setup of the sample before the lateral pull, that shows a small zone empty of grains, on the side of the sleeper, which requires some movement before the full resistance of the sample is activated.

At the microscopic scale, the interlocking phenomenon can be directly observed in the form of a concentrated force at several points points in the sample. Figures 5 and 6 show these force chains and force concentrations respectively for spherical and for cubic grains. The spherical shape reduces the number of contacts between grains, which means that the force networks that develop inside

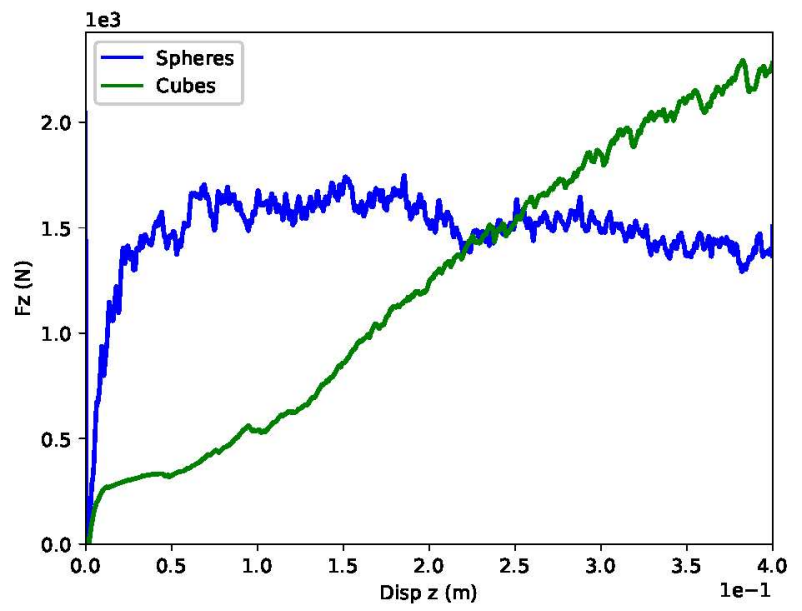

Figure 4. Lateral resistance force on sleeper for spherical and cubic grains during lateral pull test - Sliding average

the sample have less branches. This can be seen in figure 5 where a number of concentrated forces can be seen at the bottom of the sample due to the supported weight. However, when it comes to the resistance to the movement of the sleeper, the spherical grains do not present any concentrated forces opposing this movement. In the case of cubic grains (Fig. 6), the force distribution is more homogeneous, since a greater number of contact exists between grains. But in the direction of the movement of the sleeper, a number of concentrated forces can be seen developing normally to the face of the sleeper, indicating that several grains are locked into place due to their angular shape. This force concentration is not reproduced in the case of the spheres, that are allowed to slide one around the other, opposed only by the dry friction forces.

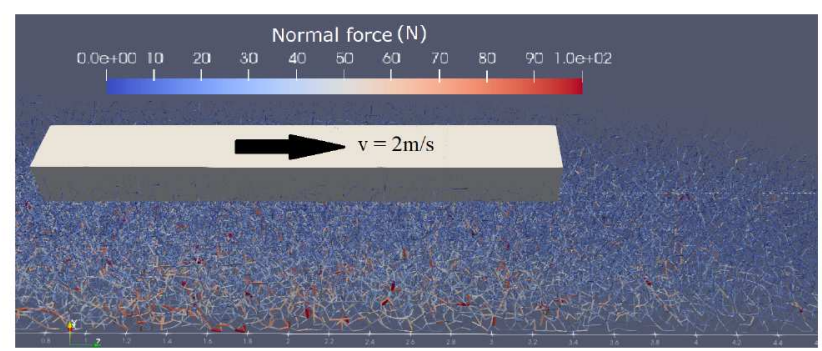

Figure 5. Normal force chain distribution in spherical grains Lateral pull test

\section{Conclusion}

This study demonstrated the importance of shape representation when modelling the lateral pull response of ballast. This was made computationally tractable by using using the BlazeDEM-GPU simulation framework. This framework exploits the parallel computing offered by GPUs to increase the efficiency of the DEM simulations significantly. This technology is used to simulate granular media, with polyhedral grains of more or less com- 


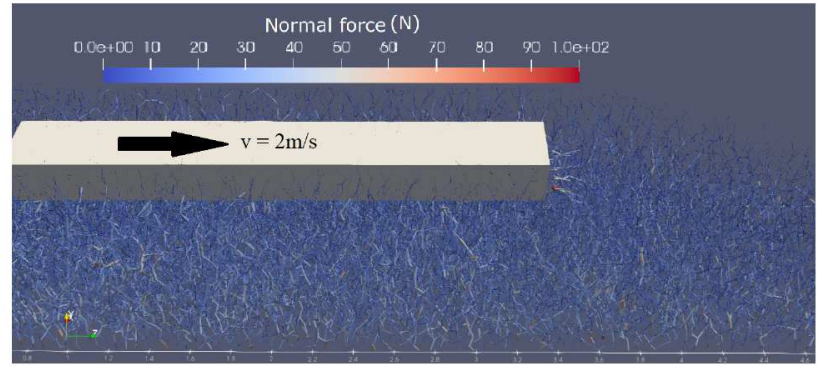

Figure 6. Normal force chain distribution in cubic grains - Lateral pull test

plexity. A database of 3D scanned ballast grains is provided by SNCF. The geometries of these grains are then treated, to eliminate any potentially useless geometrical details, as well as to control the complexity of the grains before implementation in numerical simulations. In our preliminary study, the two simulations conducted, highlight the difference between the use of spherical and cubic grains. The illustrative comparison conducted highlights phenomena that only show up when polyhedral grains are used: the frequency and magnitude of stick/slip events, as well as the distribution of force chains and its dependence on the number of contacts between grains. This becomes important when studying the behaviour of ballast grains in railway systems when zones of potential breakage and zones of breakage of asperities need to be located. The use of spheres, in that case, can artificially concentrate the force distribution in the medium or artificially reduce the strength, and to avoid these issues, a higher fidelity of grain shape is necessary to provide accurate estimations. To extend our study to identify appropriate levels of detail of actual ballast shapes when modelling lateral pull out test, a number of simulations is being conducted with ballast grain shapes of varying complexity derived from the SNCF 3D scans. The effect of the degree of details will be evaluated, and a final conclusion on the benefits of using accurate geometries when predicting the behaviour of ballast railways will be reached.

\section{References}

[1] P.A. Cundall, O.D. Strack, Géotechnique 29, 47 (1979)

[2] P.G. de Gennes, Rev. Mod. Phys. 71, S374 (1999)

[3] F. Radjaï, F. Dubois, Discrete-element modeling of granular materials (2011)

[4] S. Lobo-Guerrero, L.E. Vallejo, Granul. Matter 8, 195 (2006)

[5] M. Lu, G.R. McDowell, Geom. Geoeng. 3, 257 (2008)

[6] M. Lu, G. McDowell, Géotechnique 60, 459 (2010)

[7] N. Govender, D.N. Wilke, S. Kok, R. Els, J. Comput. Appl. Math. 270, 386 (2014)
[8] P. Pizette, N. Govender, D.N. Wilke, N.E. Abriak, DEM GPU studies of industrial scale particle simulations for granular flow civil engineering applications, in Proceedings of Powders and Grains 2017 - 8th International Conference on Micromechanics on Granular Media, EDP Sciences, 3-7 July 2017, Montpellier, France (2017)

[9] D.N. Wilke, N. Govender, P. Pizette, N.E. Abriak, Computing with non-convex Polyhedra on the GPU, in Proceedings of the International Conference on Discrete Element Methods (Springer, 2016), pp. 1371-1377

[10] N. Govender, D.N. Wilke, P. Pizette, N.E. Abriak, Appl. Math. Comput. 319, 318 (2018)

[11] P. Pizette, N. Govender, D.N. Wilke, B. Gobe, N.E. Abriak, R.K. Rajamani, 3D laser scanning technique coupled with DEM GPU simulations for railway ballasts, in Proceedings of the $V$ International Conference on Particle-Based Methods: fundamentals and applications, CIMNE, 26-28 September 2017, Hannover, Germany (2017)

[12] P.A. Cundall, A computer model for simulating progressive, large-scale movement in blocky rock system, in Proceedings of the International Symposium on Rock Mechanics, 4-6 October 1971, Nancy, France (1971)

[13] P.A. Cundall, Int. J. Rock Mech. Min. 25, 107 (1988)

[14] N. Govender, D.N. Wilke, S. Kok, SoftwareX 5, 62 (2016)

[15] C.B. Fonte, J.A. Oliveira Jr, L.C. de Almeida, DEM$C F D$ coupling: mathematical modelling and case studies using ROCKY-DEM $囚$ and ANSYS Fluent $₫$, in Proceedings of the Eleventh International Conference on CFD in the Minerals and Process Industries, CSIRO, 7-9 December 2015, Melbourne, Australia (2015)

[16] M. Hromnik, Ph.D. thesis, University of Cape Town (2013)

[17] P. Pizette, N. Govender, N.E. Abriak, D.N. Wilke, GPU DEM simulations and Experimental studies of ball milling process for various particle shapes, in Proceedings of the International Conference on Discrete Element Methods, Springer, 1-4 August 2016, Dalian, China (2016)

[18] N. Govender, D.N. Wilke, C.Y. Wu, R. Rajamani, J. Khinast, B.J. Glasser, Adv. Powder Technol. 29, 2476 (2018)

[19] S. Ravjee, S.W. Jacobsz, D.N. Wilke, N. Govender, Granul. Matter 20,68 (2018)

[20] N. Ouhbi, C. Voivret, G. Perrin, J.N. Roux, Procedia Engineer. 143, 1120 (2016)

[21] N. Ouhbi, C. Voivret, G. Perrin, J.N. Roux, Granul. Matter 19, 86 (2017) 